\title{
Comparison of vertebrate cytochrome $b$ and prepronociceptin for blood meal analyses in Culicoides
}

\section{OPEN ACCESS}

Edited by:

Elias Papadopoulos, Aristotle University, Greece

Reviewed by:

Robin James Flynn, University College Dublin, Ireland Angela Di Cesare,

University of Teramo, Italy

*Correspondence: Jérôme Depaquit,

Université de Reims Champagne-Ardenne, ANSES, SFR

Cap Santé, EA4688 - USC

"Transmission Vectorielle et Épidémiosurveillance de Maladies Parasitaires (NECPAR)", 51 rue Cognacq-Jay, Reims 51096, France jerome.depaquit@univ-reims.fr

Specialty section: This article was submitted to Parasitology, a section of the journal Frontiers in Veterinary Science

Received: 21 March 2015 Accepted: 13 May 2015 Published: 27 May 2015

Citation:

Hadj-Henni L, De Meulemeester T, Depaquit J, Noël P, Germain A Helder $R$ and Augot D (2015) Comparison of vertebrate cytochrome $b$ and prepronociceptin for blood meal analyses in Culicoides.

Front. Vet. Sci. 2:15. doi: 10.3389/fvets.2015.00015

\section{Leila Hadj-Henni ${ }^{1}$, Thibaut De Meulemeester ${ }^{2}$, Jérôme Depaquit ${ }^{1 *}$, Philippe Noël ${ }^{1}$, Adeline Germain ${ }^{1}$, Remi Helder ${ }^{3}$ and Denis Augot ${ }^{1}$}

\begin{abstract}
1 Université de Reims Champagne-Ardenne, ANSES, SFR Cap Santé, EA4688 - USC «Transmission Vectorielle et Épidémiosurveillance de Maladies Parasitaires (VECPAR)», Reims, France, ${ }^{2}$ Naturalis Biodiversity Center, Leiden, Netherlands,

${ }^{3}$ Laboratoire IAE, Université de Reims Champagne-Ardenne, Station URCA-CERFE, Boult-aux-Bois, France
\end{abstract}

To date, studies on host preferences and blood meal identification have been conducted for Culicoides species using molecular-based methods such as PCR techniques to amplify only a fragment from universal vertebrate mitochondrial genes such as cytochrome c oxidase subunit I or cytochrome b (Cyt b). The vertebrate prepronociceptin gene (PNOC) was also tested in this field. However, the choice of molecular marker to identify blood meal is critical. The objective of our study is to compare the ability of Cyt $b$ and PNOC as molecular markers for blood meal identification depending on the stage of blood meal digestion. In order to determine whether these Cyt $b$ and PNOC could provide a positive result, 565 blood-fed females of Culicoides spp were collected and morphologically identified. The samples were collected between 2012 and 2014, in two localities in France. The collection localities were near either livestock or a forest. To catch the specimens, we used UV CDC miniature light traps. PNOC sequence of donkeys (Equus asinus) was sequenced and submitted because it was missing in GenBank. Our findings emphasize that the PNOC marker is not suitable to separate closely related Equid species such as horses and donkeys. The Cyt $b$ marker was able to identify 204 more samples when compared to PNOC (99.55\% of specimens). Cyt $b$ appears to be better able to detect the origin of blood meals from females with digested blood in their abdomens. We conclude that Cyt $b$ is a good marker as it increases the accuracy of blood meal identification of engorged females containing digested blood in their abdomens. The host opportunist behavior of Culicoides, especially that of $C$. obsoletus and $C$. scoticus, the main vectors of BTV in Europe was also highlighted.

Keywords: Culicoides, blood meal, PCR, PNOC, Cyt b, France

\section{Introduction}

The study of host preferences of the native population of hematophagous Culicoides spp is critical in assessing and taking action to prevent infectious disease outbreaks. A better knowledge of this field could help identify the animal reservoirs for arboviruses. In Europe, before BTV-8 experience, bluetongue was considered an exotic disease that could be controlled by animal movement restriction and vaccination (1). Since the spread of BTV-8 in northern Europe, despite the absence of C. imicola, the main African vector of BTV, some abundant autochthonous Culicoides species have been suspected and incriminated, mainly species from Obsoletus and Pulicaris groups (2). 
More recently, in autumn 2011, an unidentified disease of livestock was reported in Germany and, the etiologic agent of this disease was identified as a novel orthobunyavirus and named Schmallenberg virus (SBV) (3). Midges of the group Obsoletus have been shown to be natural vectors for this virus (4).

To date, many studies on host preferences have been conducted for Culicoides species using serological assays (5-7). However, these techniques are time consuming and have limited sensitivity and specificity (8). Molecular-based methods were also used. PCR techniques were used to amplify only a fragment from the universal vertebrate mitochondrial genes cytochrome $c$ oxidase subunit I (COI) or cytochrome b (Cyt b) (9-13). A species-specific multiplex PCR assay was also developed $(14,15)$. The vertebrate prepronociceptin gene $(P N O C)$ was also tested $(16,17)$. However, the proportion of successful identification of blood meal origins is variable and may be affected by the quality of the samples and the laboratory protocols.

To date, there is no study to compare these molecular markers, except that of Slama et al. (17) during which the authors showed that in the PNOC-PCR sequencing methods, the sensitivity of host DNA detection was lower than found by Cyt $b$ PCR method. Here, to compare and to test the ability to identify the origin of blood meals taken from vertebrate hosts of both molecular markers, i.e., PNOC and Cyt b, we carried out our study on 565 wild-caught females of Culicoides spp trapped near livestock and forest in two localities in France. Determination of the blood meal origin was carried out through simple sequencing and Blast of the sequences in GenBank. We also compared the ability of each marker to provide interpreted sequences depending on blood digestion stage. The capacity of each marker to amplify bird DNA and to separate related animal species was also checked.

\section{Materials and Methods}

\section{Collection and Morphological Identification of Biting Midges}

In this study, 565 engorged Culicoides females were collected using UV CDC miniature light traps (John W. Hock Company, FL, USA). These traps were operated monthly in the period from 2012 to 2014, from sunset to sunrise. Three traps were positioned at a farm containing livestock (horses, sheep, cattle, and other domestic animals) as described by Lassen et al. (10) and Ninio et al. (16). The fourth trap was set in a forest area (Table 1).

All blood-fed females of Culicoides were preserved in a $95 \%$ ethanol solution until their processing. To avoid crosscontamination, every dissection was performed with single-use sterile equipment. Head, genitalia, and wings were mounted between slide and cover slip to identify the specimens morphologically according to wing pattern (18). The insect identification was conducted twice by two different persons. The species as C. obsoletus and C. scoticus are difficult to distinguish morphologically and were therefore identified according to specific morphological and morphometrical characters (19). In addition, their identification was confirmed by molecular sequencing of the mitochondrial COI gene using the primers C1-J-1718 and C1-N-2191 (20) (Table 2).

\section{Extraction of DNA for Blood Meal Analyses}

Using a conservative approach, we included all available females showing any remainder of blood in their abdomen in order to detect the maximum number of potential hosts. The color of the abdomen was observed under a magnifying glass and, if necessary, under a microscope, in order to evaluate the stage of digestion of the blood meal a visual estimation of the digestion status of Culicoides blood meals). We thus included: (i) fully engorged females $(n=220)$ with red, brownish-red, and darkred abdomens (the color of the blood meal could depend on the time that passed before the midges were transferred in ethanol, and/or the length of storage in ethanol before dissection); (ii) abdomens showing advanced signs of digestion $(n=264)$; and finally (iii) females showing any remainder of blood in their abdomen $(n=81)$ (Figure $\mathbf{1})$.

Abdomens were transferred in individual, sterilized, $1.5 \mathrm{ml}$ vials, and stored at $-20^{\circ} \mathrm{C}$. After an initial manual crushing step, the extraction was carried out using the QIamp DNA mini Kit (Qiagen, GmbH, Hilden, Germany) following the manufacturer's instructions. The DNA was eluted in a final volume of $120 \mu \mathrm{l}$ of AE buffer.

\section{Blood Meal Molecular Analyses}

The blood meal analyses were performed by sequencing the vertebrate's PNOC using the primers PNOC (F) and PNOC (R) following the PCR conditions described by Haouas et al. (21). We have also used the degenerate and universal vertebrate primers from the Cyt $b$ gene modified by Kocher et al. (22). The amplification conditions and the primer sequences are illustrated in the Table 2. PCRs for both markers were performed in a $50 \mu$ l volume using $10 \mu \mathrm{l}$ of extracted DNA solution, $50 \mathrm{pmol}$ of the primers, and Taq polymerase ( $5^{\prime}$, Germany).

Contamination was checked via the inclusion of a negative control in each run. Moreover, two positive controls were also processed in each run: DNA extracts from cattle (Bos taurus) and from Mallard ducks (Anas platyrhynchos). Mallard DNA was

TABLE 1 | Sample of blood-fed Culicoides in this study.

\begin{tabular}{|c|c|c|c|c|c|}
\hline Location & Trap & Site & Coordinates & $\begin{array}{l}\text { Predominant animal species in the vicinity of the trap } \\
\text { ( } n=\text { effective when the counting is possible) }\end{array}$ & $\begin{array}{l}\text { Number of } \\
\text { blood-fed female }\end{array}$ \\
\hline $\begin{array}{l}\text { Boult-aux-Bois } \\
\text { (Ardenne) }\end{array}$ & 1 & Forest & $\begin{array}{l}49^{\circ} 25^{\prime} 55^{\prime \prime} \mathrm{N} \\
4^{\circ} 50^{\prime} 35^{\prime \prime} \mathrm{E}\end{array}$ & $\begin{array}{l}\text { Wild boar, roe deer, red deer, horses around }(n=10) \text { (nearest horses } \\
\text { were located at around } 450 \mathrm{~m} \text { from the trap) }\end{array}$ & 91 \\
\hline \multirow[t]{3}{*}{$\begin{array}{l}\text { Louvois } \\
\text { (Marne) }\end{array}$} & 2 & Pasture & $\begin{array}{l}49^{\circ} 6^{\prime} 6^{\prime \prime} \mathrm{N} \\
4^{\circ} 7^{\prime} \mathrm{O}^{\prime \prime} \mathrm{E}\end{array}$ & $\begin{array}{l}\text { Dogs }(n=2) \text {, cats }(n=2) \text {, chickens }(n=6) \text {, geese }(n=2) \text {, ducks }(n=4) \text {, } \\
\text { pigs }(n=5) \text {, rabbits }(n=21) \text {, cattle }(n=2) \text {, goats }(n=2) \text {, horses }(n=6)\end{array}$ & 368 \\
\hline & 3 & Sheepfolds & & Sheep $(n=4)$ & 55 \\
\hline & 4 & Donkey's shelter & & Donkeys $(n=4)$, ponies $(n=2)$ & 51 \\
\hline
\end{tabular}


TABLE 2 | Primer sequences used in the molecular analyses of blood meals and Culicoides species identification.

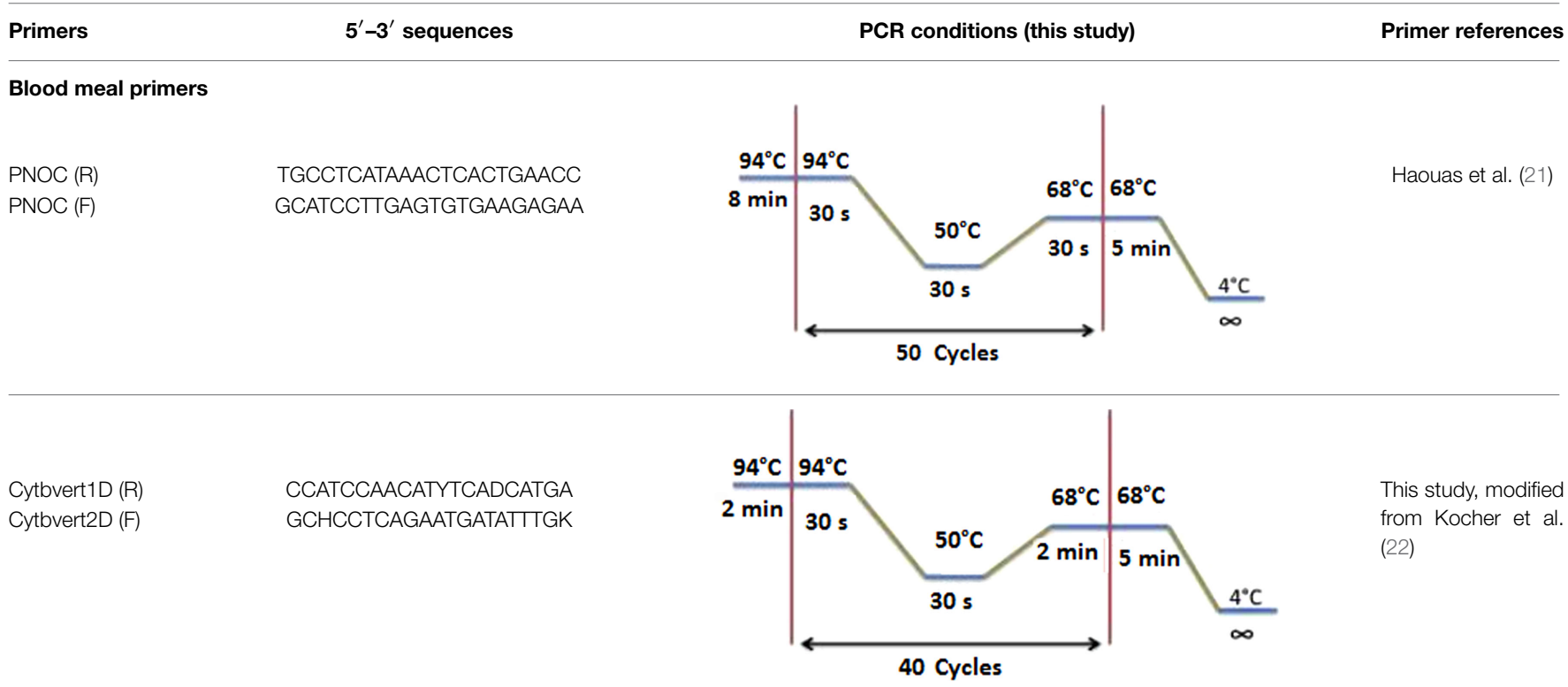

Culicoides primers

C1-N-2191 (R)

C1-J-1718 (F)
CAGGTAAAATTAAAATATAAACTTCTGG GGAGGATTTGGAAATTGATTAGT

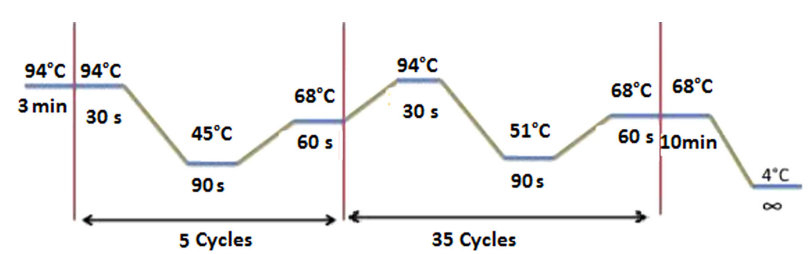

Simon et al. (20)

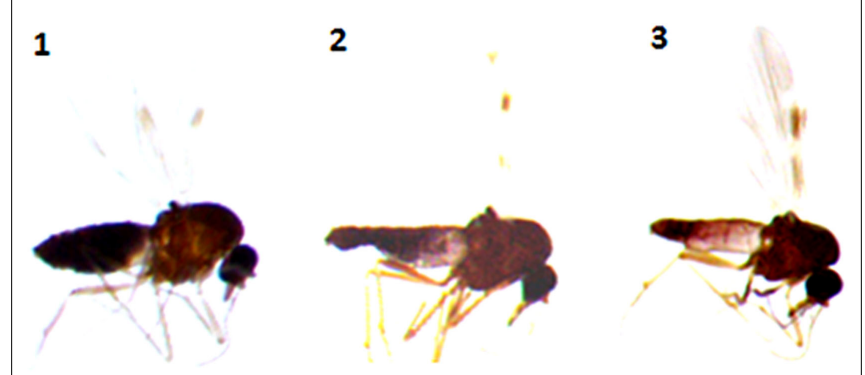

FIGURE 1 | Culicoides females with different stages of blood meal digestion. (1) Fully engorged females; (2) abdomen showing advanced signs of digestion; (3) abdomen showing any remainder of blood in their abdomen.

used to check the capacity of each marker to amplify bird DNA. The PCR products were examined using electrophoresis on a $2 \%$ agarose gel with ethidium bromide staining. PCR products were sequenced bi-directionally using the primers typically used for PCR.

\section{Sequencing and Data Analyses}

Sequences of at least $200 \mathrm{bp}$ for PNOC and $300 \mathrm{bp}$ for Cyt $b$ were compared to homologous sequences deposited in GenBank using BLAST. Blood meals were considered as identified to the species level if their sequence showed $\geq 98 \%$ homology with a sequence deposited into GenBank. Sequence editing and analyses were performed using BioEdit Sequence Alignment Editor V7.2.3 software.

\section{Statistical Analyses}

To test the efficiency of each marker to provide a positive result and to check if the two markers react in the same way at different stages of digestion ( $P N O C$ vs. $C y t b$ ), a chi-square analyses were carried out.

\section{Results}

\section{Culicoides Species Identification}

A total of 59,704 Culicoides biting midges [females (nulliparous, blood-fed, gravid, parous) and males] were collected by the four UV CDC miniature light traps used in this study during the period 2012-2014. The total sample included the following groups: 33,470 were nulliparous (56.05\%), 565 were blood-fed (0.95\%), 6173 were gravid (10.34\%), 17,988 were parous $(30.13 \%)$, and 1508 were male $(2.53 \%)$.

In the present study, the most abundantly sampled species was C. obsoletus (34.64\%), followed by C. achrayi (24.54\%), C. scoticus (14.43\%), and C. punctatus (13.2\%). Although C. lupicaris (2.06\%), C. newsteadi (1.44\%), C. furcillatus (5.77\%), C. brunnicans $(0.44 \%)$, C. pulicaris (1.44\%), C. subfasciipennis (1.88\%), C. vexans $(0.2 \%)$, and $C$. chiopterus $(0.4 \%)$ were less numerous in our sampling (Table 3). 
TABLE 3 | Origin of blood meals from blood-fed Culicoides spp.

Culicoides species ${ }^{\mathrm{a}} \quad$ Wing patterns
(number by red

blood/any remainder

of blood $^{\text {) }}$ )

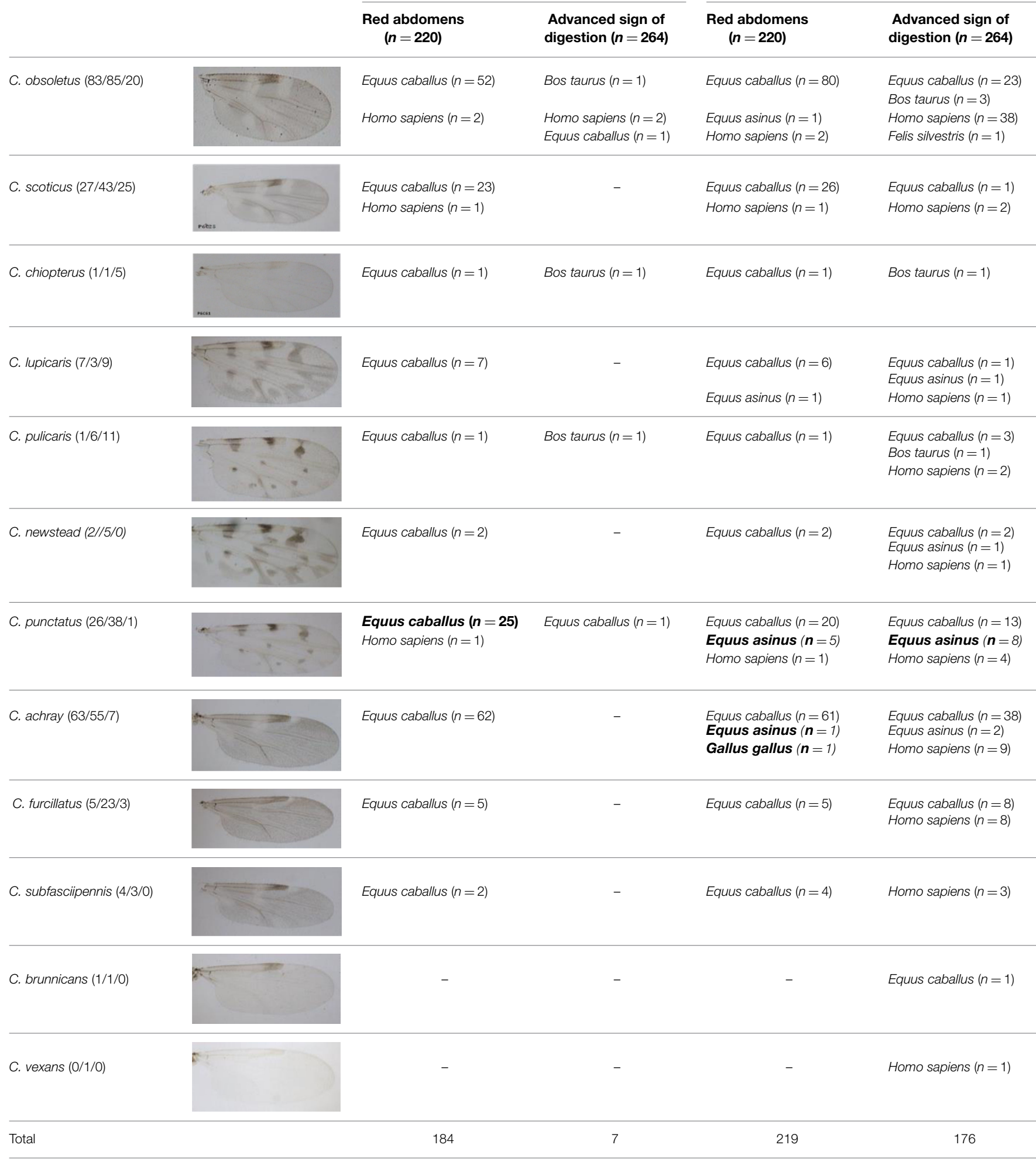

aThe same data set was tested for both markers.

${ }^{b}$ Sample with any remainder of blood $(n=81)$ are not represented in the table because they provide all a negative or not interpretable results with both markers. 


\section{Blood Meals Analyses}

From the 565 Culicoides identified as blood-fed, at least 11 different species were found to have fed on horses (73.92\% for Cyt $b$ and 95.28\% for PNOC), 5 different species on donkeys (Equus asinus), 4 different species on cattle, only 1 on a cat (Felis silvestris), and 1 on a chicken (Gallus gallus). Finally, Cyt $b$ analyses show that $19.20 \%$ of Culicoides fed on humans (Homo sapiens) vs. $4.81 \%$, the result established with PNOC (Table 3).

Analyses from $C y t b$ show that all $C$. punctatus, which were caught in Boult-aux-Bois (forest) $(n=13)$ (Table 3), are the main species that took blood meals from donkeys. Further, we observed in GenBank two haplotypes of horse sequences with a PNOC gene differing by a single base (e.g., sequences under the accession numbers XM_001493120 and AY011855) (Figure 2). We noted that the sequence of donkeys was missing. Consequently, we provided it from donkey blood following the protocol previously described (GenBank accession number KM521860). We compared the donkey blood and found it to be identical to the first haplotype of Equus caballus (accession number XM_001493120). This led us to the conclusion that the PNOC gene is unable to distinguish E. caballus sequences from those of $E$. asinus (99\% homology) whereas Cyt $b$ is able to distinguish both species.

Equally, no sequences were reported from PNOC using the Mallard DNA as opposed to the successful amplification that was reported using Cyt b. Evidence of mixed blood meals was not observed in the present study.

In our study, analyses from PNOC and Cyt $b$ genes detected blood meals of all 220 fully engorged females, 184 (83.63\%) and 219 (99.55\%), respectively. During the analyses from females showing advanced signs of blood digestion $(n=264)$, PNOC provided only seven sequences (2.65\%) whereas $C y t b$ identified 176 blood meals (66.67\%). No identification was reported from either marker on abdomens containing only traces of blood.

Statistical analyses from Cytb $\left(\chi^{2}=281.3717, \quad \mathrm{df}=2\right.$, $p<2.2 \mathrm{e}-16)$ and PNOC $\left(\chi^{2}=399.9959, \mathrm{df}=2, p<2.2 \mathrm{e}-16\right)$ show a very low $p$-value for both markers, meaning that the success of the two markers to provide a positive result is different depending the stage of digestion. Comparison of the difference in success between the two markers (PNOC vs. Cytb) provided a zero value of $p$-value $\left(\chi^{2}=1717.429, \mathrm{df}=2, p=0\right)$ meaning that markers do not react the same way facing the stage of digestion.

\section{Discussion}

In the present study, the PCR technique based on the Cyt $b$ gene of mitochondrial DNA (mtDNA) and the PNOC was selected for blood meal identification: (i) fully engorged Culicoides, (ii) Culicoides showing an advanced sign of digestion, and (iii) females showing any remainder of blood in their abdomen. The objective was to determine the ability of each marker to detect DNA from vertebrate hosts in the abdomens of Culicoides depending on the stage of blood meal digestion. Our results show that the $C y t b$ marker was more efficient (66\%) than PNOC to detect the origin of blood meals in advanced stages of digestion; this could be explained by the fact that immature erythrocytes have lost their mitochondria during the final stages of maturation, which are found in abdomens of engorged arthropods (23). Several studies have also highlighted that there is a significant negative relationship between the time since ingestion and the success of analyses $(21,24-26)$. It is interesting to note that most studies in this field include only fully engorged females. The lack of identification of blood meal origin by both markers in digested blood and in abdomens with some traces of blood can be explained by the fact that host DNA was extensively degraded during digestion. Kirsten and Gray (27) in their study on engorged Ixodes ricinus ticks, a vector of Lyme disease, explain that nested PCR amplification of PCR product could increase detection sensitivity and the limit of detection to 40 days postdigestion (27).

In the present study, no interpretable sequences were reported with both markers, perhaps due to contamination even though the samples were prepared under sterile conditions. DNA purification prior to PCR would be necessary for these samples and the use
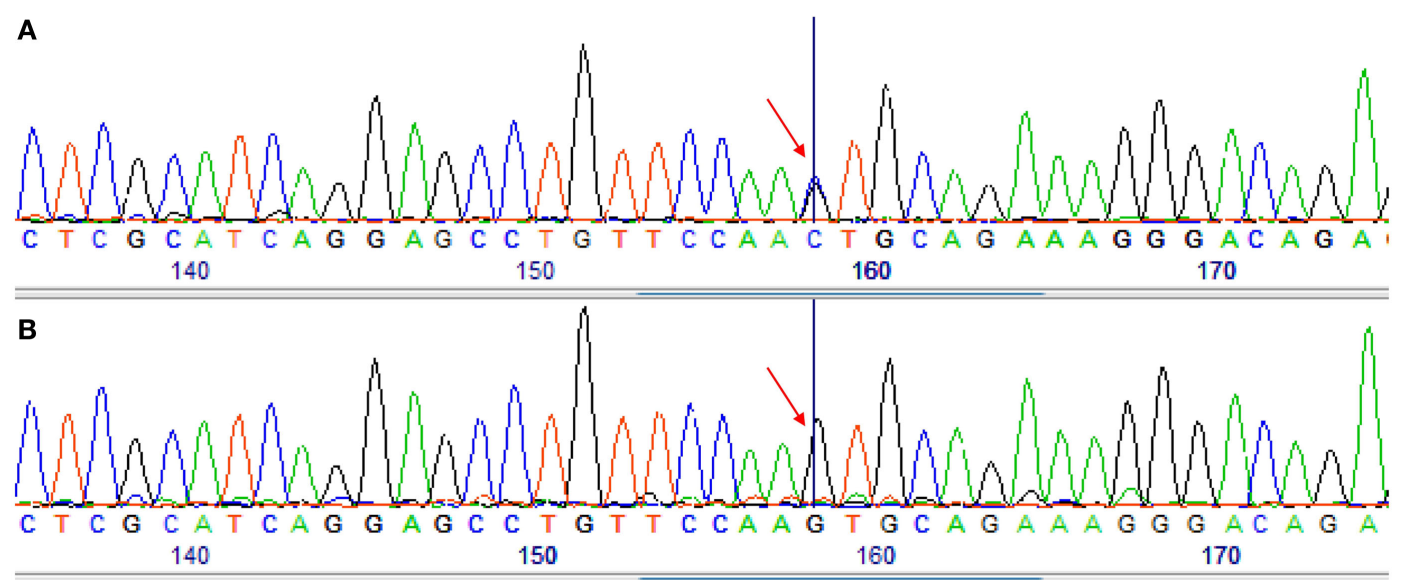

FIGURE 2 | Sequencing chromatograms obtained by sequencing of the PNOC-PCR products for two different samples. (A) A sample identified as Equus asinus by Cytb sequencing. (B) A sample identified as Equus caballus by PNOC and Cytb sequencing. Arrow shows the difference between the two samples. 
of specific-species primers could increase the yield of successful identification (14).

More than $19 \%$ of our positive samples gave sequences that matched Homo sapiens in our study. In order to check contamination, it would be better to test Culicoides provided sequences matching Homo sapiens consistently with humanspecific primers that amplify a segment of the hyper variable mitochondrial control region (28). In fact, it was not expected that primers amplifying specific segments of human mtDNA would also amplify the corresponding segments of mtDNA from other species (22).

In our study, no identification conflicts were reported between PNOC and Cyt $b$ markers except blood meals originating from donkeys and horses because the PNOC marker failed to separate donkey and horse DNA. The same findings were reported in the study of Ninio et al. (16), where no differentiation was made with PNOC between wild boars (Sus scrofa scrofa) and pigs (Sus scrofa). We conclude that PNOC is not suitable to separate closely related species such as Equidae; this marker is not a good candidate to be used alone. We confirm also the findings of Haouas et al. (21) and Slama et al. (17), in which PNOC is only suitable to detect blood meal origin from mammals but not from birds. In addition, the $P N O C$ gene underperforms in detecting blood meal origin, especially from abdomens with digested blood. Consequently, we confirm that in this type of study, the gene of choice is the Cyt $b$, because it is present in numerous copies in each cell (29). It is also known that vertebrate mtDNA can evolve faster than nuclear DNA, thus they are more polymorphic and more useful for species identification (22). The Cyt $b$ marker has also been used successfully on ticks $(27,30)$, mosquitoes $(31,32)$, tsetse flies $(33)$, sandflies (34), and Culicoides $(12,13)$. In most of these studies, Cyt $b$ has proven its discriminating power although in some studies, no differentiation has been made between domestic pigs and wild boars (Sus scrofa), sheep and mouflon (Ovis gmelini musimon), and ibex (Capra ibex) and goat (Capra aegagrus) because of their close phylogenetic heritage (14). RFLP analysis based on Cyt $b$ sequences has been limited to discriminating animals of the same genus, namely, C. elaphus and C. nippon. However, analyses of the available sequence data indicate that this problem could be resolved, because sufficient variability exists in the $C y t b$ sequence to design specific probes, which could distinguish between related species (27). In the present study, our degenerate primers discriminated E. caballus and E. asinus, two closely related species from the same genus (Equus).

In the study of Pettersson et al. (12), in order to increase the opportunity for host identification, the authors added a second widely studied gene COI for blood meal analysis for samples that did not produce results with Cyt $b$ primers. Consequently, identification of Culicoides blood meals using the Cyt $b$ gene coupled with COI gene for vertebrates is a logical application since the COI and Cyt $b$ DNA barcoding regions from more species are being sequenced and submitted to public databases. The capacity for identification of blood meals via both markers will become more and more precise.

In the present study, our sampling strategy was to use light traps close to livestock as suggested by Garros et al. (14) to maximize the capture of blood-fed females on the hosts present at study sites (wild fauna for Boult-aux-Bois and domestic fauna for Louvois). Although most studies on host preferences of Culicoides found that large mammals, specifically ruminants, were the preferred host for biting midges (Culicoides spp.) $(9-11,16)$.

However, we observed the susceptibility of horses and ponies (=E. caballus) to the attacks of Culicoides biting midge species (more than two-thirds of blood-fed Culicoides, examined with both markers, fed on horses). This observation could be expected given that horses and ponies were the most common hosts available in the vicinity of the four trap locations. In fact, every trap had horse as the most numerous blood meal host. Even the trap in the forest caught a higher number of specimens with blood meals originating from horses than any other potential hosts even though no horse was nearer than $450 \mathrm{~m}$. In addition, C. obsoletus was the most common species found to have fed on horses () suggesting that this species is prone to opportunistic feeding behavior as it is known to be attracted to horses, cattle, and sheep (9, 10, 35-38). Host-feeding behavior of Culicoides species may also be influenced by semiochemicals factors, and even shape or size of potential hosts (39-41). In the present study, no mixed blood meals were detected. Our results also suggest that other species than the putative vectors can feed on horses, including C. furcillatus, C. subfasciipennis, C. brunnicans, and C. achrayi. There are poor studies on those species maybe because is not predominant in light traps' catches (16). In our study, C. achrayi was abundant. It would also be interesting in further studies to assess the variety of hosts through diversification of trapping sites and a larger sampling. The investigation on host-feeding pattern of those species and on other species of Culicoides in general is important in understanding the epidemiology of Culicoides transmitted diseases, which affect domestic as horses and wild animals as well.

Analyses from $C y t b$ show that some blood-fed females ( $C$. punctatus) that were caught on Boult-aux-Bois took blood meals from donkeys; note that a donkey was closer than $\sim 1.3 \mathrm{~km}$ from where the blood meal was collected (trap $\mathrm{N}^{\circ} 1$, Table 1). Lassen et al. (10) collected Culicoides fed on cattle in a forest area $800 \mathrm{~m}$ from the nearest cattle; they also collected Culicoides fed on sheep $1.5 \mathrm{~km}$ from the nearest sheep. This is another example, of bloodfed biting midges traveling long distances after their blood meal. It also indicates that blood-engorged Culicoides species may engage in long-distance dispersal, engorged females were still able to cover a distance of about $200 \mathrm{~m}$ (42). This may have been caused by wind drift; indeed, it is known that midges are easily carried by the wind stream.

Unfortunately, no females fed on wild animals were found, the same observations were reported by Bartsch et al. (42), Garros et al. (14), and Ninio et al. (16). It can be assumed that biting midges prefer domestic livestock if available. In addition, as most species of Culicoides are displaying crepuscular or nocturnal behavior (43), they may prefer to bite domestic animals resting at night instead of wild animals in movement.

Since the study of blood meals is also an important component in understanding the pathogenic role of hematophagous insects, especially Culicoides, the present study indicates that the PCR direct sequencing system using universal primer sets for vertebrate $C y t b$ gene is a promising technique for blood meal 
identification especially because: (i) Cyt $b$ mtDNA has a multicopy loci (8000 copy per cell) (44) and (ii) primers used for the amplification of the PNOC gene do not recognize birds and reptiles DNA $(17,45)$. It is more judicious then to concentrate efforts to provide a single homogeneous protocol to render studies comparable. Supplementary studies on the diurnal activity of Culicoides and their breeding sites and capturing methods could contribute to the understanding of their feeding behavior and provide additional information on their role in the transmission of pathogens.

\section{Author Contributions}

LH-H, JD, TM, and DA designed this study, carried out data collection, data analysis, data interpretation, and composed the

\section{References}

1. Wilson AJ, Mellor PS. Bluetongue in Europe: past, present and future. Philos Trans R Soc Lond B Biol Sci (2009) 364(1530):2669-81. doi:10.1098/rstb.2009. 0091

2. Mellor PS, Wittmann EJ. Bluetongue virus in the Mediterranean Basin 19982001. Vet J (2002) 164(1):20-37. doi:10.1053/tvjl.2002.0713

3. Hoffmann B, Scheuch M, Höper D, Jungblut R, Holsteg M, Schirrmeier H, et al. Novel orthobunyavirus in cattle, Europe, 2011. Emerg Infect Dis (2012) 18(3):469-72. doi:10.3201/eid1803.111905

4. Rasmussen LD, Kristensen B, Kirkeby C, Rasmussen TB, Belsham GJ, Bødker R. Culicoides as vectors of Schmallenberg virus. Emerg Infect Dis (2012) 18(7):1204-6. doi:10.3201/eid1807.120385

5. Murray MD. The identification of blood meals in biting midges, (Culicoides: Ceratopogonidae). Ann Trop Med Parasitol (1970) 64(1):115-22.

6. Blackwell A, Mordue AJ, Mordue W. Identification of blood meals of the Scottish biting midge, Culicoides impunctatus, by indirect enzyme-linked immunosorbent assay (ELISA). Med Vet Entomol (1994) 8(1):20-4. doi:10.1111/ j.1365-2915.1994.tb00378.x

7. Blackwell A, Brown M, Mordue W. The use of an enhanced ELISA method for the identification of Culicoides blood meals in host-preference studies. Med Vet Entomol (1995) 9(2):214-8. doi:10.1111/j.1365-2915.1995.tb00183.x

8. Önder Ö, Shao W, Lam H, Brisson D. Tracking the sources of blood meals of parasitic arthropods using shotgun proteomics and unidentified tandem mass spectral libraries. Nat Protoc (2014) 9(4):842-50. doi:10.1038/nprot.2014.048

9. Calvo JH, Berzal B, Calvete C, Miranda MA, Estrada R, Lucientes J. Host feeding patterns of Culicoides species (Diptera: Ceratopogonidae) within the Picos de Europa National Park in Northern Spain. Bull Entomol Res (2012) 102(6):692-7. doi:10.1017/S0007485312000284

10. Lassen SB, Nielsen SA, Kristensen M. Identity and diversity of blood meal hosts of biting midges (Diptera: Ceratopogonidae: Culicoides latreille) in Denmark. Parasit Vectors (2012) 5(1):143. doi:10.1186/1756-3305-5-143

11. Martínez-de la Puente J, Martínez J, Ferraguti M, Morales-de la Nuez A, Castro N, Figuerola J. Genetic characterization and molecular identification of the bloodmeal sources of the potential bluetongue vector Culicoides obsoletus in the Canary Islands, Spain. Parasit Vectors (2012) 5:147. doi:10.1186/ 1756-3305-5-147

12. Pettersson E, Bensch S, Ander M, Chirico J, Sigvald R, Ignell R. Molecular identification of blood meals and species composition in Culicoides biting midges. Med Vet Entomol (2013) 27(1):104-12. doi:10.1111/j.1365-2915.2012. 01038.x

13. Santiago-Alarcon D, Havelka P, Pineda E, Segelbacher G, Schaefer HM. Urban forests as hubs for novel zoonosis: blood meal analysis, seasonal variation in Culicoides (Diptera: Ceratopogonidae) vectors, and avian haemosporidians. Parasitology (2013) 140(14):1799-810. doi:10.1017/S0031182013001285

14. Garros C, Gardès L, Allène X, Rakotoarivony I, Viennet E, Rossi S, et al. Adaptation of a species-specific multiplex PCR assay for the identification of blood meal source in Culicoides (Ceratopogonidae: Diptera): applications on paper. PN, RH, and AG carried out data collection and data analysis.

\section{Acknowledgments}

This work is funded by the CPER project "Culichamp" and by ANSES. The authors thank Stavana Strutz for proofreading this manuscript. TM was supported in part by "STEP - Status and Trends of European Pollinators" (EC FP7 grant no. 244090) and by SUPER-B COST ACTION FA 1305. The study was also funded by an internal Applied-Oriented Research Grant from Naturalis Biodiversity Center. We are grateful to Modeste De Meulemeester and Véronique Dubois for help in donkey blood acquisition. The authors would like to thank the reviewers for their constructive comments which helped to improve the manuscript.

palaearctic biting midge species, vectors of orbiviruses. Infect Genet Evol (2011) 11(5):1103-10. doi:10.1016/j.meegid.2011.04.002

15. Ayllón T, Nijhof AM, Weiher W, Bauer B, Allène X, Clausen PH. Feeding behaviour of Culicoides Spp. (Diptera: Ceratopogonidae) on cattle and sheep in Northeast Germany. Parasit Vectors (2014) 7(1):34. doi:10.1186/ 1756-3305-7-34

16. Ninio C, Augot D, Delecolle JC, Dufour B, Depaquit J. Contribution to the knowledge of Culicoides (Diptera: Ceratopogonidae) host preferences in France. Parasitol Res (2011) 108(3):657-63. doi:10.1007/s00436-010-2110-9

17. Slama D, Haouas N, Mezhoud H, Babba H, Chaker E. Blood meal analysis of Culicoides (Diptera: Ceratopogonidae) in Central Tunisia. PLoS One (2015) 10(3):e0120528. doi:10.1371/journal.pone. 0120528

18. Delécolle J-C. Nouvelle contribution à létude systématique et iconographique des espèces du genre Culicoides, (Diptéra): (Cératopogonidae) du Nord-Est de la France. Strasbourg: Delécolle (J-C) (1985).

19. Augot D, Sauvage F, Jouet D, Simphal E, Veuille M, Couloux A, et al. Discrimination of Culicoides obsoletus and Culicoides scoticus, potential bluetongue vectors, by morphometrical and mitochondrial cytochrome oxidase subunit I analysis. Infect Genet Evol (2010) 10(5):629-37. doi:10.1016/j.meegid. 2010.03.016

20. Simon C, Francesco F, Andrew B, Bernie C, Hong L, et Paul F. Evolution, weighting, and phylogenetic utility of mitochondrial gene sequences and a compilation of conserved polymerase chain reaction primers. Annals of the Entomological Society of America (1994) 87(6):651-701.

21. Haouas N, Pesson B, Boudabous R, Dedet JP, Babba H, Ravel C. Development of a molecular tool for the identification of Leishmania reservoir hosts by blood meal analysis in the insect vectors. Am J Trop Med Hyg (2007) 77(6): 1054-9.

22. Kocher TD, Thomas WK, Meyer A, Edwards SV, Paabo S, Villablanca FX, et al. Dynamics of mitochondrial DNA evolution in animals: amplification and sequencing with conserved primers. Proc Natl Acad Sci U S A (1989) 86(16):6196-200. doi:10.1073/pnas.86.16.6196

23. Tyler DD. The Mitochondrion in Health and Disease. edn. 1. New York:VCH (1992).

24. Mukabana WR, Takken W, Knols BG. Analysis of arthropod blood meals using molecular genetic markers. Trends Parasitol (2002) 18(11):505-9. doi:10.1016/ S1471-4922(02)02364-4

25. Oshaghi MA, Chavshin AR, Vatandoost H, Yaaghoobi F, Mohtarami F, Noorjah N. Effects of post-ingestion and physical conditions on PCR amplification of host blood meal DNA in mosquitoes. Exp Parasitol (2006) 112(4):232-6. doi:10.1016/j.exppara.2005.11.008

26. Martínez-de la Puente J, Ruiz S, Soriguer R, Figuerola J. Effect of blood meal digestion and DNA extraction protocol on the success of blood meal source determination in the malaria vector Anopheles atroparvus. Malar J (2013) 12(1):109. doi:10.1186/1475-2875-12-109

27. Kirstein F, Gray JS. A molecular marker for the identification of the zoonotic reservoirs of Lyme borreliosis by analysis of the blood meal in its European vector Ixodes ricinus. Appl Environ Microbiol (1996) 62(11):4060-5. 
28. Morin PA, Moore JJ, Chakraborty R, Jin L, Goodall J, Woodruff DS. Kin selection, social structure, gene flow, and the evolution of chimpanzees. Science (1994) 265(5176):1193-201. doi:10.1126/science.7915048

29. Townzen JS, Brower AVZ, Judd DD. Identification of mosquito blood meals using mitochondrial cytochrome oxidase subunit I and cytochrome $b$ gene sequences. Med Vet Entomol (2008) 22(4):386-93. doi:10.1111/j.1365-2915. 2008.00760.x

30. Wodecka B, Rymaszewska A, Skotarczak B. Host and pathogen DNA identification in blood meals of nymphal Ixodes ricinus ticks from forest parks and rural forests of Poland. Exp Appl Acarol (2014) 62(4):543-55. doi:10.1007/ s10493-013-9763-x

31. Lee JH, Hassan H, Hill G, Cupp EW, Higazi TB, Mitchell CJ, et al. Identification of mosquito avian-derived blood meals by polymerase chain reactionheteroduplex analysis. Am J Trop Med Hyg (2002) 66(5):599-604.

32. Kent RJ, Norris DE. Identification of mammalian blood meals in mosquitoes by a multiplexed polymerase chain reaction targeting cytochrome $b$. Am J Trop Med Hyg (2005) 73(2):336-42.

33. Steuber S, Abdel-Rady A, Clausen PH. PCR-RFLP analysis: a promising technique for host species identification of blood meals from tsetse flies (Diptera: Glossinidae). Parasitol Res (2005) 97(3):247-54. doi:10.1007/ s00436-005-1410-y

34. Laskay ÜA, Burg J, Kaleta EJ, Vilcins IM, Telford III SR, Barbour AG, et al. Development of a host blood meal database: de novo sequencing of hemoglobin from nine small mammals using mass spectrometry. Biol Chem (2012) 393(3):195-201. doi:10.1515/hsz-2011-0196

35. Townley P, Baker KP, Quinn PJ. Preferential landing and engorging sites of Culicoides species landing on a horse in Ireland. Equine Vet J (1984) 16(2):117-20. doi:10.1111/j.2042-3306.1984.tb01876.x

36. Carpenter S, Szmaragd C, Barber J, Labuschagne K, Gubbins S, Mellor P. An assessment of Culicoides surveillance techniques in Northern Europe: have we underestimated a potential bluetongue virus vector? J Appl Ecol (2008) 45(4):1237-45.doi:10.1111/j.1365-2664.2008.01511.x

37. Gerry AC, Sarto i Monteys V, Moreno Vidal JO, Francino O, Mullens BA. Biting rates of Culicoides midges (Diptera: Ceratopogonidae) on sheep in Northeastern Spain in relation to midge capture using UV light and carbon dioxide-baited traps. J Med Entomol (2009) 46(3):615-24. doi:10.1603/033.046.0329

38. Viennet E, Garros C, Gardès L, Rakotoarivony I, Allène X, Lancelot R, et al. Host preferences of palaearctic Culicoides biting midges: implications for transmission of orbiviruses. Med Vet Entomol (2013) 27(3):255-66. doi:10.1111/ j.1365-2915.2012.01042.x

39. Humphreys JG, Turner EC. Blood-feeding activity of female Culicoides (Diptera: Ceratopogonidae). J Med Entomol (1973) 10(1):79-83. doi:10.1093/ jmedent/10.1.79

40. Raich T, Jacobson M, Holbrook F, Babion R, Blair C, Beaty B. Culicoides variipennis (Diptera: Ceratopogonidae) host selection in Colorado. J Med Entomol (1997) 34(2):247-9. doi:10.1093/jmedent/34.2.247

41. Logan JG, Seal NJ, Cook JI, Stanczyk NM, Birkett MA, Clark SJ, et al. Identification of human-derived volatile chemicals that interfere with attraction of the Scottish biting midge and their potential use as repellents. J Med Entomol (2009) 46(2):208-19. doi:10.1603/033.046.0205

42. Bartsch S, Bauer B, Wiemann A, Clausen PH, Steuber S. Feeding patterns of biting midges of the Culicoides obsoletus and Culicoides pulicaris groups on selected farms in Brandenburg, Germany. Parasitol Res (2009) 105(2):373-80. doi:10.1007/s00436-009-1408-y

43. Mellor PS, Boorman J, Baylis M. Culicoides biting midges: their role as arbovirus vectors. Annu Rev Entomol (2000) 45(1):307-40. doi:10.1146/annurev.ento.45. 1.307

44. Lah EF, Ahamad M, Haron MS, Ming HT. Establishment of a molecular tool for blood meal identification in Malaysia. Asian Pac J Trop Biomed (2012) 2(3):223-7. doi:10.1016/S2221-1691(12)60046-X

45. Murphy WJ, Eizirik E, O’Brien SJ, Madsen O, Scally M, Douady CJ, et al. Resolution of the early placental mammal radiation using Bayesian phylogenetics. Science (2001) 294(5550):2348-51.

Conflict of Interest Statement: The authors declare that the research was conducted in the absence of any commercial or financial relationships that could be construed as a potential conflict of interest.

Copyright (C) 2015 Hadj-Henni, De Meulemeester, Depaquit, Noël, Germain, Helder and Augot. This is an open-access article distributed under the terms of the Creative Commons Attribution License (CC BY). The use, distribution or reproduction in other forums is permitted, provided the original author(s) or licensor are credited and that the original publication in this journal is cited, in accordance with accepted academic practice. No use, distribution or reproduction is permitted which does not comply with these terms. 